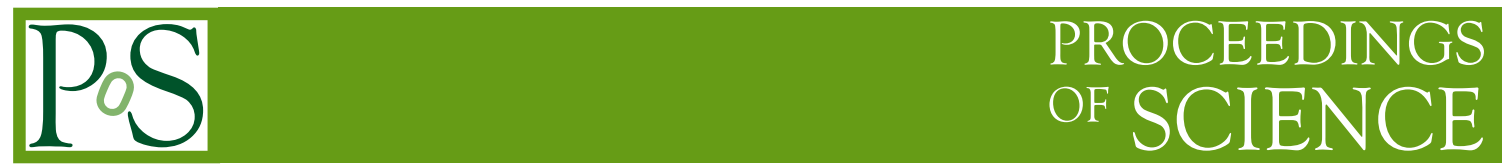

\title{
NA61/SHINE at the CERN SPS
}

\section{Andras Laszlo* for the NA61 Collaboration}

KFKI Research Institute for Particle and Nuclear Physics, Budapest, Hungary

E-mail: laszloa@rmki.kfki.hu

\section{The NA61 Collaboration:}

N. Abgrall ${ }^{23}$, A. Aduszkiewicz ${ }^{24}$, B. Andrieu ${ }^{11}$, T. Anticic ${ }^{13}$, N. Antoniou ${ }^{18}$, A. G. Asryan ${ }^{15}$, B. Baatar ${ }^{9}$, A. Blondel ${ }^{23}$, J. Blumer $^{5}$, L. Boldizsar ${ }^{10}$, A. Bravar ${ }^{23}$, J. Brzychczyk $^{8}$,

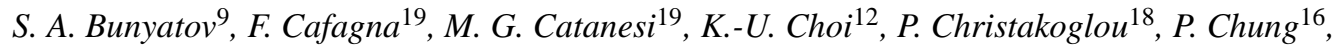
J. Cleymans ${ }^{1}$, D. A. Derkach ${ }^{15}$, F. Diakonos ${ }^{18}$, W. Dominik ${ }^{24}$, J. Dumarchez $^{11}$, R. Engel ${ }^{5}$, A. Ereditato ${ }^{21}$, G. A. Feofilov ${ }^{15}$, Z. Fodor ${ }^{10}$, M. Gazdzicki ${ }^{17,22}$, M. Golubeva $^{6}$, K. Grebieszkow ${ }^{25}$, F. Guber ${ }^{6}$, A. Haungs ${ }^{5}$, M. Hess ${ }^{21}$, S. Igolkin ${ }^{15}$, A. S. Ivanov ${ }^{15}$, A. Ivashkin ${ }^{6}$, K. Kadija ${ }^{13}$, R. Karabowicz ${ }^{8}$, N. Katrynska ${ }^{8}$, D. Kielczewska ${ }^{24}$, D. Kikola ${ }^{25}$, J.-H. Kim ${ }^{12}$, T. Kobayashi ${ }^{7}$, V. I. Kolesnikov ${ }^{9}$, D. Kolev ${ }^{4}$, R. S. Kolevatov ${ }^{15}$, V. P. Kondratiev ${ }^{15}$, A. Kurepin ${ }^{6}$, R. Lacey ${ }^{16}$, A. Laszlo ${ }^{10}$, S. Lehmann ${ }^{21}$, B. Lungwitz ${ }^{22}$, V. V. Lyubushkin ${ }^{9}$, A. Maevskaya ${ }^{6}$, Z. Majka ${ }^{8}$, A. I. Malakhov ${ }^{9}$, A. Marchionni ${ }^{2}$, M. Di Marco ${ }^{23}$, V. Matveev ${ }^{6}$, G. L. Melkumov ${ }^{9}$, A. Meregaglia ${ }^{2}$, M. Messina ${ }^{21}$, C. Meurer ${ }^{5}$, P.Mijakowski $^{14}$, M. Mitrovski $^{22}$, T. Montaruli ${ }^{18, \#}$, St. Mrówczyński ${ }^{17}$, S. Murphy ${ }^{23}$, T. Nakadaira ${ }^{7}$, P. A. Naumenko ${ }^{15}$, V. Nikolic ${ }^{13}$, T. Palczewski $^{14}$, G. Palla ${ }^{10}$, A. D. Panagiotou ${ }^{18}$, W. Peryt ${ }^{25}$, A. Petridis ${ }^{18}$, R. Planeta ${ }^{8}$, J. Pluta ${ }^{25}$, B. A. Popov ${ }^{9}$, M. Posiadala ${ }^{24}$, P. Przewlocki ${ }^{14}$, E. Radicioni ${ }^{19}$,W. Rauch ${ }^{3}$, R. Renford $^{22}$, D. Röhrich $^{20}$, E. Rondio ${ }^{14}$, B. Rossi ${ }^{21}$, M. Roth ${ }^{5}$, A. Rubbia ${ }^{2}$, M. Rybczynski ${ }^{17}$, A. Sadovsky ${ }^{6}$, K. Sakashita ${ }^{7}$, T. Schuster ${ }^{22}$, T. Sekiguchi ${ }^{7}$, P. Seyboth ${ }^{17}$, K. Shileev ${ }^{6}$, A. N. Sissakian ${ }^{9}$, E. Skrzypczak ${ }^{24}$, M. Slodkowski ${ }^{25}$, A. S. Sorin ${ }^{9}$, P. Staszel ${ }^{8}$, G. Stefanek ${ }^{17}$, J. Stepaniak ${ }^{14}$, C. Strabel ${ }^{2}$, H. Stroebele ${ }^{22}$, T. Susa ${ }^{13}$, I. Szentpetery ${ }^{10}$, M. Szuba $^{25}$, A. Taranenko ${ }^{16}$, R. Tsenov ${ }^{4}$, M. Unger ${ }^{5}$, M. Vassiliou ${ }^{18}$, V. V. Vechernin ${ }^{15}$, G. Vesztergombi ${ }^{10}$, Z. Wlodarczyk ${ }^{17}$, A. Wojtaszek ${ }^{17}$, J.-G. Yi $^{12}$, I. - K. Yoo ${ }^{12}$ 


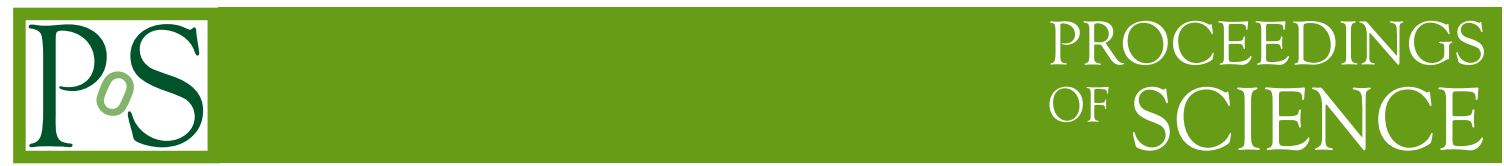

${ }^{1}$ Cape Town University, Cape Town, South Africa

${ }^{2}$ ETH, Zurich, Switzerland

${ }^{3}$ Fachhochschule Frankfurt, Frankfurt, Germany

${ }^{4}$ Faculty of Physics, University of Sofia, Sofia, Bulgaria

${ }^{5}$ Forschungszentrum Karlsruhe, Karlsruhe, Germany

${ }^{6}$ Institute for Nuclear Research, Moscow, Russia

${ }^{7}$ Institute for Particle and Nuclear Studies, KEK, Tsukuba, Japan

${ }^{8}$ Jagellionian University, Cracow, Poland

${ }^{9}$ Joint Institute for Nuclear Research, Dubna, Russia

${ }^{10}$ KFKI Research Institute for Particle and Nuclear Physics, Budapest, Hungary

${ }^{11}$ LPNHE, University of Paris VI and VII, Paris, France

${ }^{12}$ Pusan National University, Pusan, Republic of Korea

${ }^{13}$ Rudjer Boskovic Institute, Zagreb, Croatia

${ }^{14}$ Soltan Institute for Nuclear Studies, Warsaw, Poland

${ }^{15}$ St. Petersburg State University, St. Petersburg, Russia

${ }^{16}$ State University of New York, Stony Brook, USA

${ }^{17}$ Świ etokrzyska Academy, Kielce, Poland

${ }^{18}$ University of Athens, Athens, Greece

${ }^{19}$ University of Bari and INFN, Bari, Italy

${ }^{20}$ University of Bergen, Bergen, Norway

${ }^{21}$ University of Bern, Bern, Switzerland

${ }^{22}$ University of Frankfurt, Frankfurt, Germany

${ }^{23}$ University of Geneva, Geneva, Switzerland

${ }^{24}$ University of Warsaw, Warsaw, Poland

${ }^{25}$ Warsaw University of Technology, Warsaw, Poland

${ }^{\#}$ Now at University of Wisconsin, Madison, USA.

Status of the new experimental program to study hadron production in hadron-nucleus and nucleus-nucleus collisions at the CERN SPS will be presented. In particular, a detailed physics motivation and experimental strategy will be given for the part of the program related to the physics of strongly interacting matter: search for the critical point of strongly interacting matter, study properties of the onset of deconfinement, and high $p_{T}$ measurements in $\mathrm{p}+\mathrm{p}$ and $\mathrm{p}+\mathrm{A}$ interactions. The planned measurements for the neutrino $\mathrm{T} 2 \mathrm{~K}$ and cosmic-ray experiments will also be discussed.

Critical Point and Onset of Deconfinement - 4th International Workshop

July 9 - 13, 2007

Darmstadt, Germany

* Speaker. 


\section{Introduction}

The NA61/SHINE [1] is a new fixed-target experiment at the CERN SPS accelerator, based on the upgraded setup of the NA49 apparatus. In particular, the most expensive components are inherited from the NA49. These are the two superconducting magnets, the four large volume TPCs and the two ToF walls.

The physics programme of NA61 is the systematic measurement of hadron production in proton-proton, proton-nucleus, hadron-nucleus, and nucleus-nucleus collisions. This comprehensive study mainly concerns the following objectives.

1. Search for the critical point by an energy - system size scan.

2. Study the properties of the onset of deconfinement by the energy - system size scan.

3. To establish, together with the RHIC results, the energy dependence of the nuclear modification factor.

4. To record hadron-nucleus reference spectra for the T2K neutrino experiment, and for the Pierre Auger Observatory and KASCADE cosmic-ray experiments.

The detector upgrades shall also be discussed in the light of the necessities, posed by the physics goals.

\section{Physics goals}

\subsection{Search for the critical point and onset of deconfinement}

As predicted by lattice QCD calculations (see e.g. [2]), the phase diagram of the strongly interacting matter admits a phase border of 1-st order phase transition on the temperature - baryochemical potential, which has a critical endpoint. According to the calculations, this critical endpoint may be located in the energy range, accessible at the CERN SPS.

Although the temperature $(T)$ and baryochemical potential $\left(\mu_{B}\right)$ are not directly measurable quantities, the $T-\mu_{B}$ coordinates of the freeze-out points of nuclear reactions can be brought into one-to-one correspondence with the energy $(E)$ and system size $(A)$ of the nuclear collisions (see e.g. [3]). Therefore, the $T-\mu_{B}$ coordinates of the freeze-out points may be scanned via a systematic $E-A$ scan. When the phase evolution of a given collision passes near the critical endpoint, the increase of scaled variance $(\omega)$ of multiplicity distribution and transverse momentum fluctuations measure $\left(\Phi_{p_{T}}\right)$ are expected (see e.g. [4]). Thus, the critical endpoint may be discovered by looking at the energy and system size dependence of the multiplicity and transverse momentum fluctuations. A performance simulation of the detector for measuring transverse momentum fluctuations is shown in Figure 1. The $\Phi_{p_{T}}$ measure calculated within the UrQMD model in the NA61 acceptance is shown for the reactions planned to be taken by NA61 (left panel). In the right panel additional fluctuations $(10 \mathrm{MeV} / \mathrm{c})$ as expected in the vicinity of the critical point are added to a point for $\mathrm{S}+\mathrm{S}$ collisions at $80 \mathrm{~A} \mathrm{GeV} \mathrm{[5].}$

The detector upgrades and reactions to be studied are determined by the necessity of the reduction of the background fluctuations, in particular caused by the fluctuations of the number of 

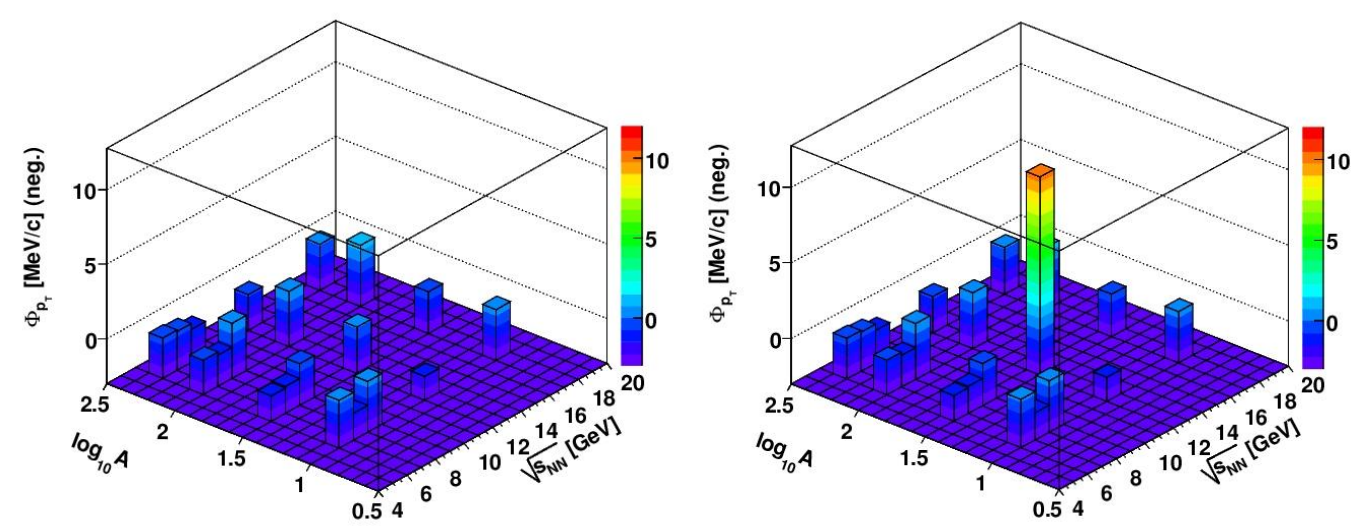

Figure 1: Performance simulation for measuring transverse momentum fluctuations. UrQMD data (left) and artificial fluctuations added to this background (right).

participant nucleons. As shown in Figure 2 even for a fixed number of the projectile participants (by a calorimetric measurement of the number of projectile spectators) the number of target participants fluctuates [6]. These fluctuations which constitute a significant background in a search for fluctuation signals of the critical point and the onset of deconfinement can be suppressed by selecting very central collisions of identical nuclei. The present centrality trigger facility, the VCAL (a downstream calorimeter), is measuring the energy, carried by the projectile spectators: the collision is central if the spectator energy is small. However, the energy resolution of the VCAL is not good enough: a new projectile spectator energy measurement facility, the Projectile Spectator Detector (PSD), is being built, with much better resolution, to enable a precise selection of very central collisions.

An other source of background for multiplicity fluctuation measurements is the contamination by spiralling low-energy knock-on electrons ( $\delta$-electrons). To minimize this contribution, a Helium beam pipe will be introduced in the beam-line along the sensitive TPC volumes.

The planed systematic scan in energy and system size will allow to study the system size dependence of the anomalies in hadron production observed by NA49 [7] in central $\mathrm{Pb}+\mathrm{Pb}$ collisions at about 30A GeV. These anomalies were predicted for the onset of deconfinement [8] and their further understanding requires new NA61 data.

\subsection{Study high transverse momentum particle spectra}

A very interesting phenomenon, discovered by RHIC experiments at $\sqrt{s_{N N}}=200 \mathrm{GeV}$ collision energy is the reduction of high transverse momentum particle production in nuclear collisions relative to elementary collisions (see e.g. [9]), when assuming scaling of particle spectra by the number of binary collisions. This phenomenon is referred to as 'high $p_{T}$ particle suppression', and is usually interpreted as the manifestation of the parton energy loss in the formed strongly interacting matter. Study on the energy dependence of the suppression phenomenon needed for its further understanding. The idea is that if the collision energy is low enough that this strongly interacting matter is not formed, the high transverse momentum particle suppression should disappear. 


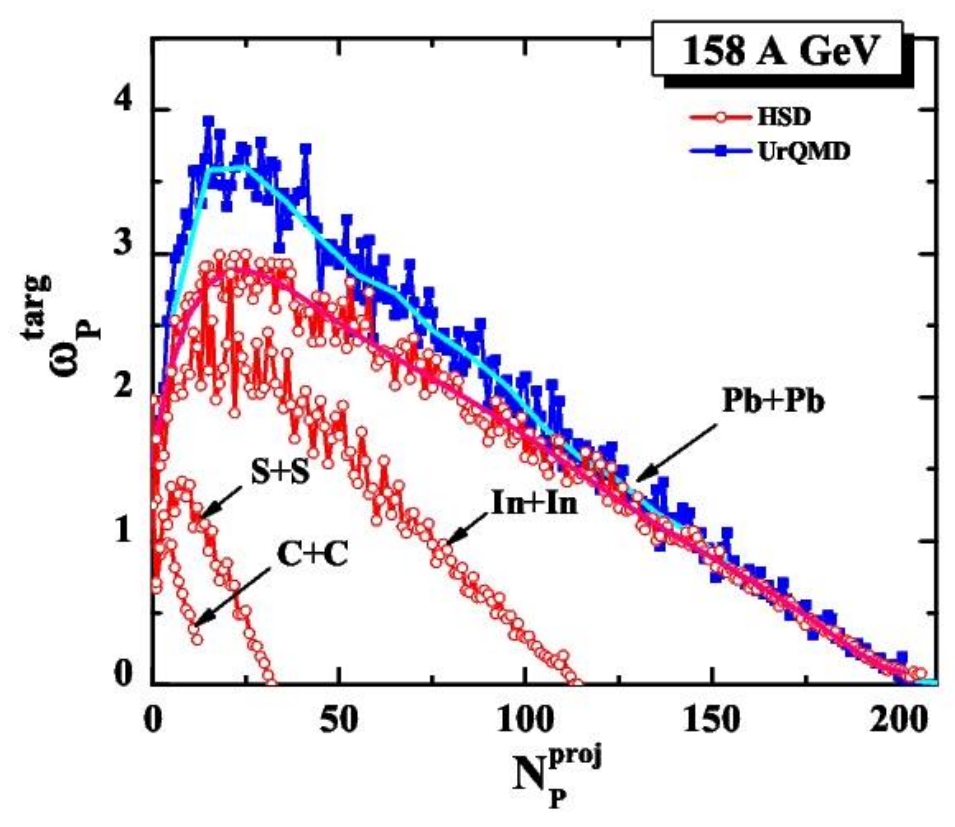

Figure 2: Fluctuation of the number of target participants, as a function of projectile participants.

The particle suppression in a reaction $\mathrm{A}+\mathrm{B}$ relative to that of $\mathrm{p}+\mathrm{p}$ is measured by the nuclear modification factor $R_{A+B}^{B C}=\frac{1}{\left\langle N_{B C}\right\rangle(A+B)} \cdot \frac{\text { Yield }(A+B)}{\operatorname{Yield}(p+p)}$, where $\left\langle N_{B C}\right\rangle(A+B)$ is the average number of binary collisions in the $\mathrm{A}+\mathrm{B}$ reaction. (By the use of $\left\langle N_{B C}\right\rangle$, it is implicitly assumed that the particle spectra, without any nuclear effects, should scale with the number of binary collisions.)

In Figure 3, a preliminary $R_{A A}$ of $\pi^{ \pm}$production at $\sqrt{s_{N N}}=17.3 \mathrm{GeV}$ is shown, based on [10] and [11] (left panel), and the published $R_{A A}$ result at $\sqrt{s_{N N}}=200 \mathrm{GeV}$ of $[12,13]$ is compared to it (right panel). (The $\mathrm{p}+\mathrm{W} / \mathrm{p}+\mathrm{p}$ curve at $\sqrt{S_{N N}}=19.4 \mathrm{GeV}$ was taken from [14].) It is seen that the NA49 curve stops at $p_{T} \geq 2 \mathrm{GeV} / \mathrm{c}$, therefore the existence or non-existence of a suppression at higher $p_{T}$ is not clear from the present data. The accessible $p_{T}$ range is limited by the available $\mathrm{p}+\mathrm{p}$ statistics.

Unfortunately, the available world-data on $\pi^{ \pm}$production in $\mathrm{p}+\mathrm{p}$ does not cover our energy region. Furthermore, due to the closeness of the kinematic border of the available momentum space, the shape of the $p_{T}$ spectra admits a rapid change from convex to concave at the SPS energy range, which makes an interpolation quite unsafe. This is shown in the left panel of Figure 4, together with a compilation of the world-data [15]. The red line marks the shape change from convex to concave with increasing beam energy. In the right panel of Figure 4, the currently available high $p_{T}$ $\pi^{ \pm}$statistics of NA49 is shown for the $5 \%$ most central $\mathrm{Pb}+\mathrm{Pb}$ collisions as well as $\mathrm{p}+\mathrm{Pb}$ and $\mathrm{p}+\mathrm{p}$ interactions at $158 \mathrm{AGeV}$ beam energy $\left(\sqrt{s_{N N}}=17.3 \mathrm{GeV}\right)$. It is seen, that the central $\mathrm{Pb}+\mathrm{Pb}$ data extend up to $p_{T}=4.5 \mathrm{GeV}$, while the $\mathrm{p}+\mathrm{Pb}$ and $\mathrm{p}+\mathrm{p}$ spectra stop at about $2.5 \mathrm{GeV} / \mathrm{c}$. To estimate the necessary statistics, needed to be recorded by NA61, the $\mathrm{Pb}+\mathrm{Pb}$ spectrum shape was used for extrapolation.

To accomplish the planned high statistics $\mathrm{p}+\mathrm{p}$ and $\mathrm{p}+\mathrm{Pb}$ measurements, the TPC readout sys- 


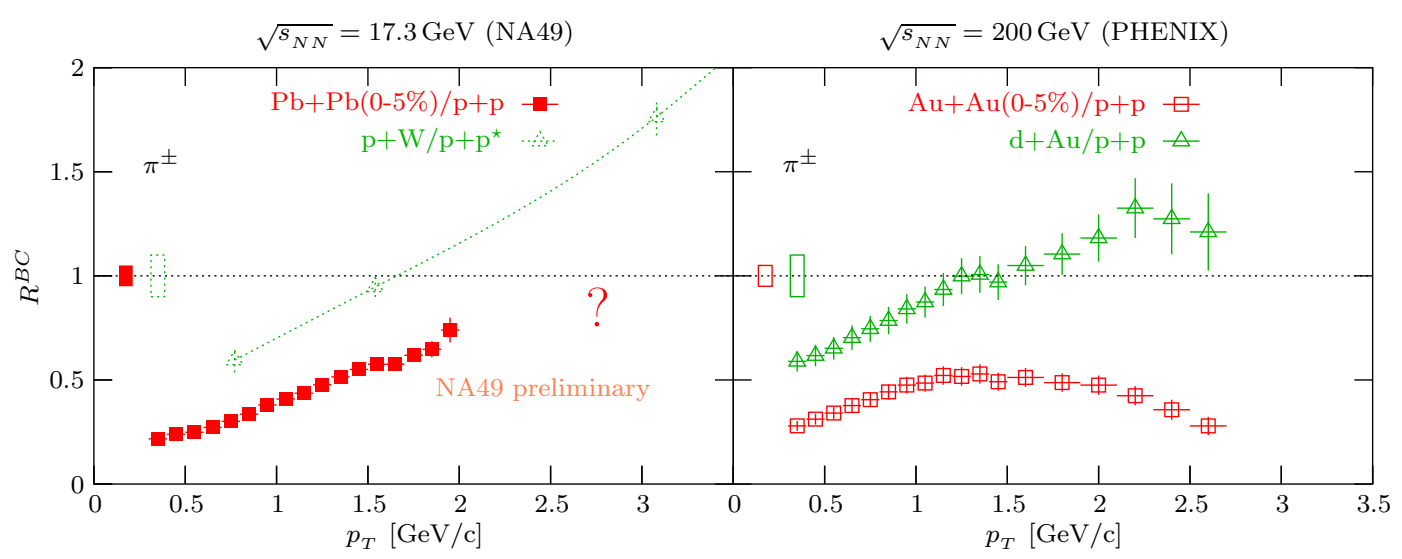

Figure 3: Nuclear modification factor $R^{B C}$ for $\pi^{ \pm}$mesons in $\mathrm{p}+\mathrm{A}$ and $\mathrm{A}+\mathrm{A}$ interactions at the top SPS and RHIC energies. The rectangles show systematic uncertainty. The $p+W / p+p$ curve was measured at $\sqrt{s_{N N}}=19.4 \mathrm{GeV}[14]$.
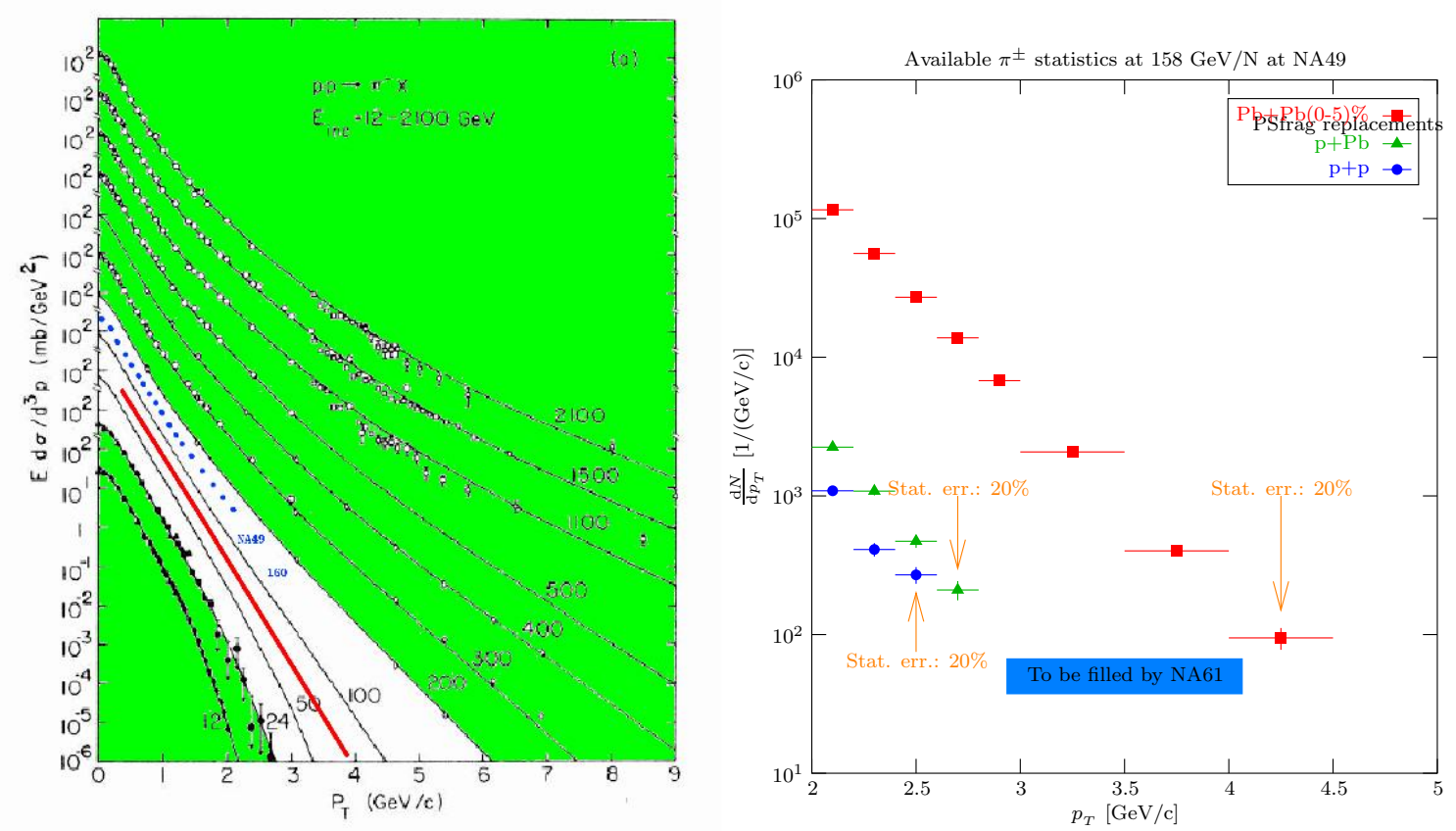

Figure 4: Left: the available $p+p$ data on $\pi^{ \pm}$production at our energy range, together with a parameterization (from [15]). The red line shows a change in spectrum shape. As can be seen: there are no data in the nearby energies, which makes the use of parameterization unsafe. Right: currently available high $p_{T} \pi^{ \pm}$ statistics at midrapidity for $\mathrm{Pb}+\mathrm{Pb}, \mathrm{p}+\mathrm{Pb}$ and $\mathrm{p}+\mathrm{p}$ reactions at $\sqrt{s_{N N}}=17.3 \mathrm{GeV}$.

tem needs to be upgraded, to be able to record events at a higher rate.

\subsection{Reference spectra for $\mathrm{T} 2 \mathrm{~K}$ and cosmic-ray experiments}

T2K [16] is a neutrino oscillation experiment at JPARC, which produces neutrino beams indi- 
rectly by $p(30,40,50 \mathrm{GeV})+C \rightarrow \pi, K+X$ reactions, and subsequent decay of pions and Kaons. The neutrinos are detected $295 \mathrm{~km}$ away, in the Super Kamiokande (SK) detector. The setup of the experiment is outlined in Figure 5. The method of the $v_{\mu} \leftrightarrow v_{e}$ oscillation measurement is based on the so-called far-to-near ratio, $R$, of the neutrino fluxes in the far (SK) and the near (ND) detectors. To predict $R$ with a required precision, initial $\pi, K$ production cross-sections in $\mathrm{p}+\mathrm{C}$ interactions at 30,40 and $50 \mathrm{GeV}$ should be measured. This will be done by NA61/SHINE experiment using a $1 \mathrm{~cm}\left(2 \% \lambda_{\text {int }}\right)$ graphite target and a $90 \mathrm{~cm}$ long T2K replica target.

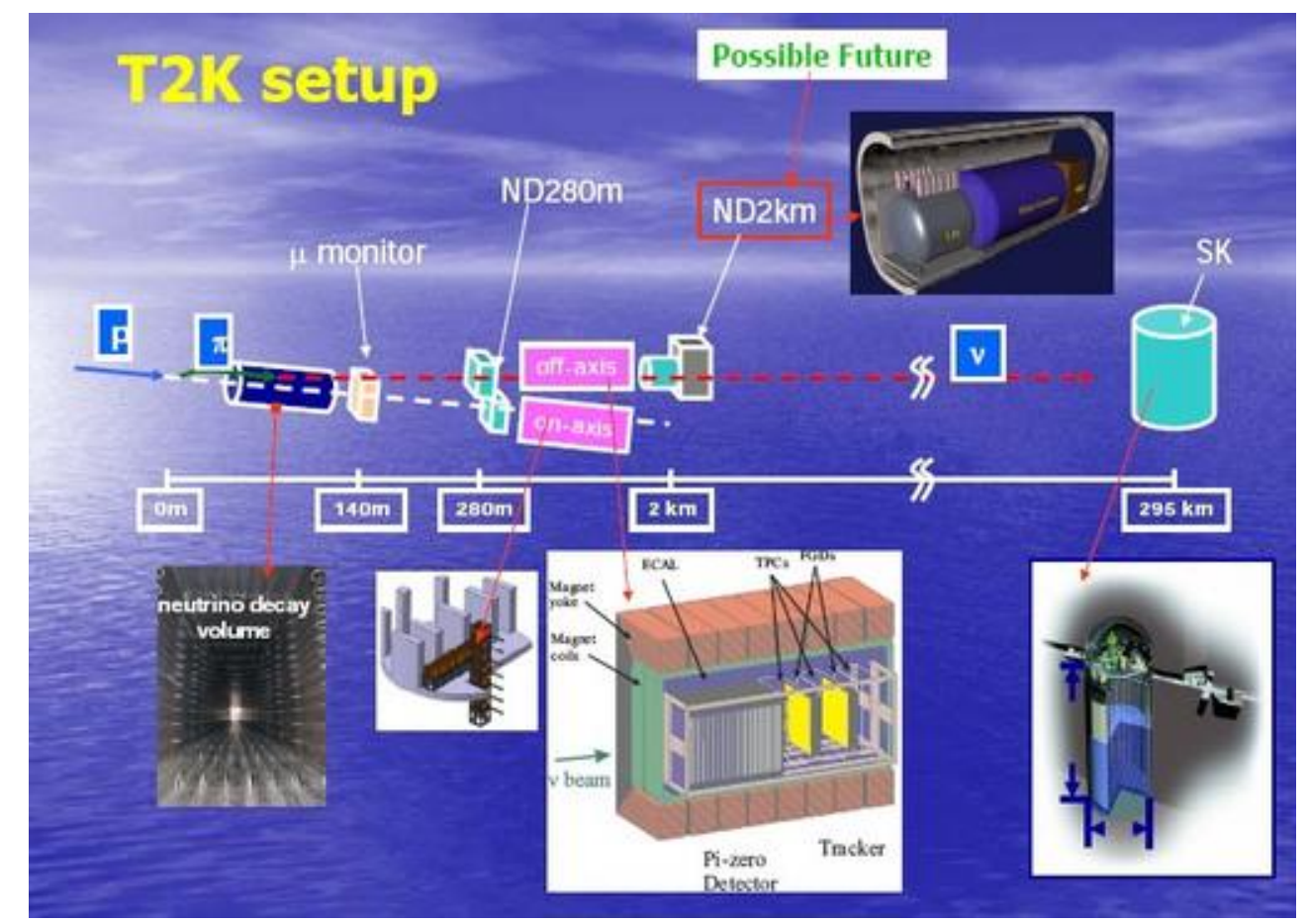

Figure 5: The outline of the $\mathrm{T} 2 \mathrm{~K}$ experiment. The produced neutrino beam is detected at the Super Kamiokande, $295 \mathrm{~km}$ away from the neutrino beam production volume.

Cosmic-ray experiments are based on detection of extensive air showers by large lateral coverage ground detector arrays. The particle type and energy of the incident particle is then reconstructed by the simulation of the air shower. These simulations are most sensitive to $\mu^{ \pm}$production in cascades, which is related to the $p, \pi+C \rightarrow \pi, K+X$ production processes at SPS energies. ${ }^{1}$ The $\mu^{ \pm}$-s are produced by the weak decay of the $\pi, K$ particles. By the precise measurement of these production cross-sections, the model dependence of the extensive air shower simulations can be largely reduced. Some measurements of the NA61/SHINE are dedicated for this purpose.

For the $\mathrm{T} 2 \mathrm{~K}$ and cosmic-ray runs, the extension of the forward acceptance for particle identification is needed. Therefore, a new forward ToF wall for the NA61 setup is being built.

\footnotetext{
${ }^{1}$ Such reactions occur when shower particles hit the nuclei of the air. A large fraction of muons detected by the ground detectors originates from decays of pions and Kaons produced in collisions at the CERN SPS energies.
} 


\section{NA61/SHINE, the upgraded NA49 detector}

\subsection{Detector setup}

The necessary detector upgrades, motivated by the physics goals, are shown in Figure 6. Due to its excellent performance, the NA49 TPC tracking system will be used in NA61. The most important performance parameters are listed below.

1. Large acceptance: $\approx 50 \%$ at $p_{T} \leq 2.5 \mathrm{GeV} / \mathrm{c}$.

2. Precise momentum measurement: $\Delta(p) / p^{2} \approx 10^{-4}(\mathrm{GeV} / \mathrm{c})^{-1}$.

3. High tracking efficiency: $\geq 95 \%$.

4. Good particle identification: ToF resolution, $\sigma(t) \approx 60 \mathrm{ps,}$ $\frac{\mathrm{d} E}{\mathrm{~d} x}$ resolution, $\sigma\left(\frac{\mathrm{d} E}{\mathrm{~d} x}\right) / \frac{\mathrm{d} E}{\mathrm{~d} x} \approx 5 \%$, invariant mass resolution, $\sigma(m) \approx 5 \mathrm{MeV}$.

The planned main upgrades are the followings.

1. New spectator calorimeter, the PSD, instead of the VCAL. The resolution to be achieved is $\frac{\sigma(E)}{E} \approx \frac{0.5}{\sqrt{E /(1 \mathrm{GeV})}}(\times 5$ improvement $)$, and the uniformity to be achieved is non-uniformity $<5 \%(\times 20$ improvement $)$.

(Motivated by the search for the critical point and onset of deconfinement.)

2. Installation of a Helium beam-pipe to reduce $\delta$-electron contamination in the sensitive volumes ( $\times 10$ improvement).

(Motivated by the search for the critical point and onset of deconfinement.)

3. Forward ToF wall (for particle identification in $p<3 \mathrm{GeV} / \mathrm{c}$ and $\theta<400 \mathrm{mrad}$ region). (Motivated by the T2K measurements.)

4. New TPC readout with about $100 \mathrm{~Hz}$ readout frequency $(\times 10$ improvement).

(Motivated by all physics goals.)

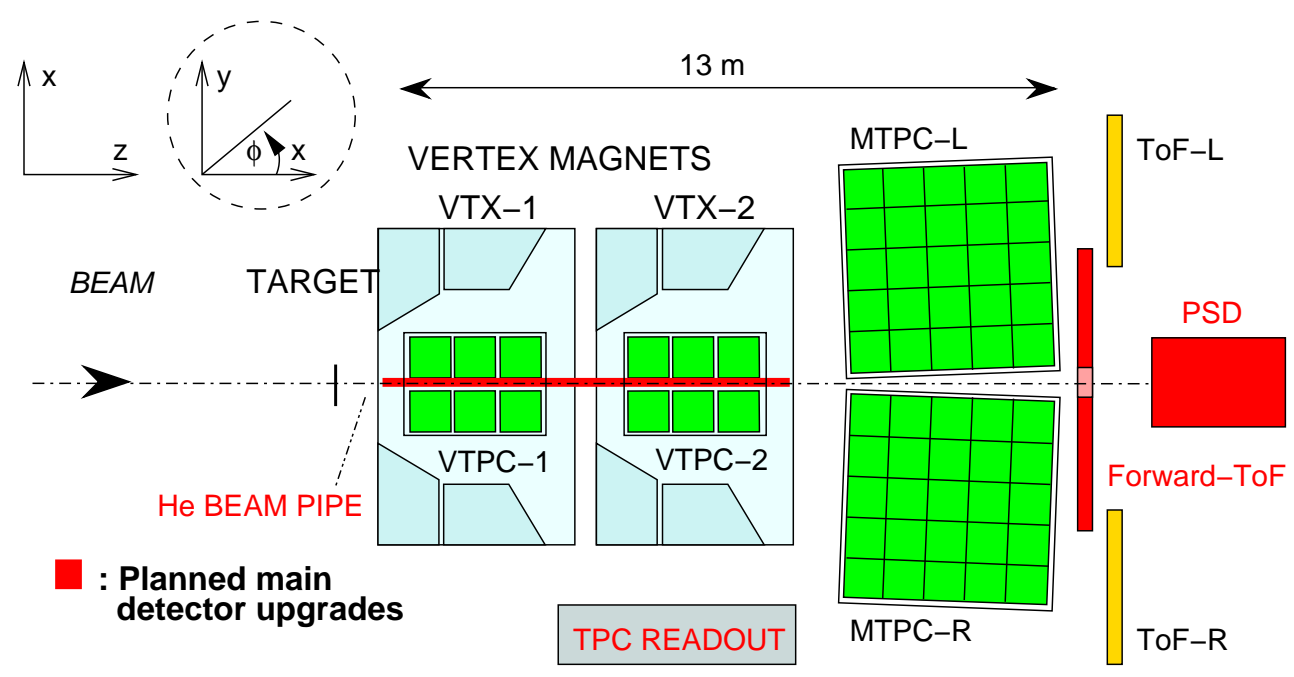

Figure 6: The set-up of the NA61/SHINE experiment. The main detector detector upgrades are shown together with the original devices of NA49. 


\subsection{Status and planning}

The beam request of NA61 is summarized in Table 1, together with the current recommendation and approval status assigned by the SPS Committee and CERN Research Board.

\begin{tabular}{llllll} 
Beam & Energy $[\mathrm{A} \mathrm{GeV}]$ & Year & Days & Physics & Status \\
\hline \hline$p$ & 30 & 2007 & 30 & T2K, CR & Approved \\
$p$ & $30,40,50$ & 2008 & 14 & T2K, CR & Recommended \\
$\pi^{-}$ & 158,350 & 2008 & 3 & CR & Recommended \\
$p$ & 158 & 2008 & 28 & high $p_{T}$ & Recommended \\
$S$ & $10,20,30,40,80,158$ & 2009 & 30 & CP\&OoD & Recommended \\
$p$ & $10,20,30,40,80,158$ & 2009 & 30 & CP\&OoD & Recommended \\
In & $10,20,30,40,80,158$ & 2010 & 30 & CP\&OoD & To be discussed \\
$p$ & 158 & 2010 & 30 & high $p_{T}$ & To be discussed \\
$C$ & $10,20,30,40,80,158$ & 2011 & 30 & CP\&OoD & To be discussed \\
$p$ & $10,20,30,40,80,158$ & 2011 & 30 & CP\&OoD & To be discussed \\
\hline
\end{tabular}

Table 1: The beam request of the NA61/SHINE experiment. Abbreviations: CP - search for Critical Point; OoD - study the Onset of Deconfinement; T2K - supplementary spectra for the T2K experiment; CR measurements for cosmic-ray physics; high $p_{T}-\mathrm{p}+\mathrm{p}$ and $\mathrm{p}+\mathrm{Pb}$ reference spectra for nuclear modification factors.

For the approval procedure of the NA61 experiment, the following documents are the most relevant: Expression of Interest [17], Letter of Intent [18], Status Report [19], Proposal [20], Addendum-1 [21] and Addendeum-2 [22].

\section{Summary}

The experiment NA61/SHINE has a great discovery potential to find the critical point of strongly interacting matter, if exists.

Important measurements of the nuclear modification factor at the top SPS energy can be performed and the system size dependence of the effects related to the onset of deconfinement can be studied.

The NA61 experiment at the CERN SPS is complementary to other projects on nucleusnucleus collision currently developed at FAIR, JINR, BNL and CERN LHC. It will provide the necessary input for the neutrino and cosmic-ray experiments at KEK, FZK.

\section{Acknowledgments}

This work was supported by the US Department of Energy, the Virtual Institute VI-146 of Helmholtz Gemeinschaft, Germany, the Korea Science \& Engineering Foundation (R01-2005-00010334-0), the Hungarian Scientific Research Fund (OTKA 68506), the Polish Ministry of Science and Higher Education (N N202 3956 33). 


\section{References}

[1] The NA61/SHINE homepage [http://na61,web.cern.ch].

[2] Z. Fodor, S. D. Katz, JHEP 0203 (2002) 014.

[3] F. Becattini, J. Manninen, M. Gazdzicki, Phys. Rev. C73 (2006) 044905.

[4] M. Stephanov, K. Rajagopal, E. Shuryak, Phys. Rev. D60 (1999) 114028.

[5] K. Grebieszkow (NA61 Collaboration), private communication.

[6] V. P. Konchakovski et al, Phys. Rev. C73 (2006) 034902.

[7] S. V. Afanasiev et al (the NA49 Collaboration), Phys. Rev. C66 (2002) 054902.

[8] M. Gazdzicki, M. I. Gorenstein, Acta Phys. Polon. B30 (1999) 2705.

[9] S. S. Adler et al (the PHENIX Collaboration), Phys. Rev. Lett. 91 (2003) 072303.

[10] A. Laszlo, High transverse momentum identified charged particle yields in $158 \mathrm{GeV} / n u c l e o n \mathrm{~Pb}+\mathrm{Pb}$ collisions, NA49 technical note (2007).

[11] C. Alt et al (the NA49 Collaboration), Eur. Phys. J. C45 (2006) 342.

[12] S. S. Adler et al (the PHENIX Collaboration), Phys. Rev. C69 (2004) 034909.

[13] S. S. Adler et al (the PHENIX Collaboration), Phys. Rev. C74 (2006) 024904.

[14] Antreasyan et al, Rhys. Rev. D19 (1979) 764.

[15] E. W. Beier et al, Phys. Rev. D19 (1978) 2235.

[16] The T2K homepage [http://jnusrv01.kek.jp/public/t2k].

[17] J. Bartke et al (the NA61 Collaboration), A new experimental programme with nuclei and proton beams at the CERN SPS, NA49-future expression of interest (2003), CERN-SPSC-2003-031, SPSC-EOI-001.

[18] N. Antoniou et al (the NA61 Collaboration), Study of hadron production in collisions of protons and nuclei at the CERN SPS, NA49-future letter of intent (2006), CERN-SPSC-2006-001, SPSC-I-235.

[19] N. Antoniou et al (the NA61 Collaboration), Report from tests of the NA49 experimental facility and the NA49-future detector prototypes, NA49-future status report (2006), CERN-SPSC-2006-023, SPSC-SR-010.

[20] N. Antoniou et al (the NA61 Collaboration), Study of hadron production in hadron-nucleus and nucleus-nucleus collisions at the CERN SPS, NA49-future proposal (2006), CERN-SPSC-2006-034, SPSC-P-330.

[21] N. Antoniou et al (the NA61 Collaboration), Additional information requested in the proposal review process, Addendum-1 to the NA49-future proposal (2007), CERN-SPSC-2007-004, SPSC-P-330.

[22] N. Antoniou et al (the NA61 Collaboration), Further information requested in the proposal review process, Addendum-2 to the NA49-future proposal (2007), CERN-SPSC-2007-019, SPSC-P-330. 